\title{
Dificuldades e Facilidades do processo educativo desenvolvido por enfermeiros às pessoas com estomias
}

\author{
What nurses find difficult and helpful when educating people with ostomies \\ Dificultades y facilidades del proceso educativo desarrollado por enfermeras para personas con estomas
}

\author{
Vanessa Cristina Maurício' $\bullet$, Norma Valéria Dantas de Oliveira Souza" $\odot$, Carlos Eduardo Maurícioll' $\odot$, \\ Carolina Cabral Pereira da Costa" $\odot$, Marcia de Souza Silva" $\odot$, Anna Beatryz Marques Roque" $\odot$
}

IInstituto Nacional de Traumatologia e Ortopedia, Rio de Janeiro, RJ, Brasil; "Universidade do Estado do Rio de Janeiro, Rio de Janeiro, RJ, Brasil

\begin{abstract}
RESUMO
Objetivo: descrever e analisar as dificuldades e facilidades percebidas por enfermeiros para implementação do processo educativo dirigido às pessoas com estomia. Método: estudo qualitativo e exploratório, apoiada no materialismo histórico e no método dialético. A coleta ocorreu em um Centro de Reabilitação no Rio de Janeiro, com seis enfermeiros, por meio da triangulação de métodos (observação participante, entrevista e análise de registros de enfermagem), entre dezembro de 2014 e março de 2015. Resultados: os fatores dificultadores do processo educativo foram: estrutura física inadequada, escassez de recursos financeiro e humano. Além disso, apreendeu-se déficit na formação e qualificação dos enfermeiros. Os fatores facilitadores incluíram: fornecimento de equipamentos e adjuvantes para as pessoas com estomia e criação de grupos de apoio para esta clientela. Conclusão: concluiu-se que existem contradições e discrepâncias na execução do processo educativo relacionadas à organização laboral, à formação e aos aspectos legais.

Descritores: Enfermagem; Estomas Cirúrgicos. Reabilitação; Educação em Saúde.
\end{abstract}

\section{ABSTRACT}

Objective: to describe and analyze what nurses perceive to be difficult and simple to implement in the process of educating people with ostomies Method: this qualitative, exploratory study was supported by historical materialism and the dialectical method. Data were collected from six nurses at a Rehabilitation Center in Rio de Janeiro, using a triangulation of methods (participant observation, interview, and analysis of nursing records), between December 2014 and March 2015. Results: the factors that hampered the educational process were inappropriate physical structure, and scarcity of financial and human resources. There was also found to be a deficit in the nurses' training and qualification. Enabling factors included: provision of equipment and adjuvants for people with ostomies, and creation of support groups for this clientele. Conclusion: it was concluded that execution of the educational process suffers from contradictions and discrepancies relating to work organization, training, and legal aspects.

Descriptors: Nursing; Surgical Stomas. Rehabilitation; Health Education.

\section{RESUMEN}

Objetivo: describir y analizar lo que las enfermeras perciben como difícil y simple de implementar en el proceso de educación de las personas con ostomías Método: este estudio cualitativo, exploratorio, se sustenta en el materialismo histórico y el método dialéctico. Se recolectaron datos de seis enfermeras en un Centro de Rehabilitación de Río de Janeiro, mediante una triangulación de métodos (observación participante, entrevista y análisis de registros de enfermería), entre diciembre de 2014 y marzo de 2015. Resultados: los factores que obstaculizaron el proceso educativo fueron estructura física inadecuada y escasez de recursos financieros y humanos. También se constató un déficit en la formación y calificación de las enfermeras. Los factores habilitantes incluyeron: provisión de equipo y adyuvantes para personas con ostomías y creación de grupos de apoyo para esta clientela. Conclusión: se concluyó que la ejecución del proceso educativo adolece de contradicciones y discrepancias relacionadas con la organización del trabajo, la formación y los aspectos legales.

Descriptores: Enfermería; Estomas Quirúrgicos. Rehabilitación; Educación en Salud.

\section{INTRODUÇÃO}

O objeto deste estudo trata das dificuldades e facilidades percebidas pelos enfermeiros para o desenvolvimento do processo educativo às pessoas com estomia. O termo estomia significa exteriorização cirúrgica de parte de um órgão oco, como, por exemplo, de algum trecho do aparelho digestório, respiratório e urinário, mantendo uma comunicação com o meio externo. Tal exteriorização é realizada quando há necessidade de desviar, temporária ou definitivamente, o trânsito normal das eliminações vesico-intestinais, ar e/ou alimentação ${ }^{1}$.

A confecção de uma estomia resulta em impactos significativos no funcionamento corporal, na autoimagem, na sexualidade, na possibilidade de lazer e de trabalho, entre outras dimensões da vida. Portanto, estar com uma estomia é uma situação complexa, sobretudo devido às repercussões biopsicossociais que dela advém². 
Neste sentido, o enfermeiro precisa adotar uma conduta holística, um cuidado integral, correlacionando conhecimentos técnico-científicos, éticos e humanísticos, além de desenvolver seu processo de trabalho em adição com outros membros da equipe de saúde, a fim de atender as necessidades multifacetadas das pessoas com estomias ${ }^{3}$.

A atuação do profissional de enfermagem, neste contexto assistencial, envolve a prestação do cuidado direto, atividades gerenciais, de pesquisa e ensino. Sobre o ensino, tal perspectiva do cuidado volta-se para formação e qualificação de recursos humanos ou para a educação de pessoas e familiares em situação de estomia, cuja prática envolve a orientação para o autocuidado ${ }^{3}$.

Neste âmbito, a orientação às pessoas com estomia é relevante, pois em tal situação há um desconhecimento sobre o manejo dos equipamentos coletores ou de outras tecnologias; existe um estranhamento sobre as reações e funcionamento do corpo; verificam-se dificuldades com vestuário, higienização corporal, com a sexualidade, enfim, com atividades cotidianas. Por conseguinte, se estas pessoas não forem adequadamente orientadas, há o potencial para ocorrência de elevada morbi-mortalidade ${ }^{2}$.

Este estudo justifica-se devido ao aumento crescente de pessoas com estomia no Brasil e no mundo, uma vez que se esperam 41.000 novos casos de câncer do cólon e reto no contexto nacional para cada ano do triênio de 2020-2022, e estes tipos de cânceres contribuem para a realização de uma estomias ${ }^{4}$. Além disso, entende-se que ao investigar o processo educativo de enfermeiros voltados para pessoas com estomias, é possível identificar déficits e potencialidades que podem ser corrigidos e fortalecidos, respectivamente, possibilitando um melhor e mais amplo atendimento as demandas destas pessoas.

A partir desta contextualização, selecionou-se como questão norteadora do estudo: quais são as facilidades e as dificuldades para que o enfermeiro desenvolva o processo educativo dirigido às pessoas com estomia?

Para responder tal questão, elaboraram-se os seguintes objetivos: descrever e analisar as dificuldades e facilidades percebidas por enfermeiros para implementação do processo educativo dirigido às pessoas com estomia.

\section{REVISÃO DE LITERATURA}

A presença de uma estomia determina mudanças radicais na vida das pessoas, tanto físicas quanto psicossociais, destacando-se alterações na imagem corporal ${ }^{5}$, dificuldades no uso e manejo de tecnologias de suporte a qualidade de vida, ocorrência de complicações no estoma e pele periestoma, mudanças nos hábitos alimentares e de higiene. Tal situação propicia autoestima diminuída, sexualidade comprometida, afastamento do trabalho, estigmatização e isolamento social ${ }^{6}$

A capacidade de enfrentamento diante do impacto de tais transformações está atrelada a um processo terapêutico que envolve apoio psicológico, educativo e de orientações, o qual é fornecido às pessoas com estomia pela equipe multidisciplinar de saúde, ajudado pelos familiares e amigos. Nesta perspectiva, reforça-se a importância dos enfermeiros no processo de orientação de pessoas com estomias, abrangendo ações integrais, voltadas para a realidade e necessidades individuais, favorecendo uma melhoria da qualidade de vida 6 .

O processo educativo, que permeia a ação de orientar, construído por meio da interação entre as pessoas, é um instrumento dinâmico de socialização de saberes, podendo contribuir para a autonomia no agir, possibilitando aos envolvidos tornarem-se sujeitos ativos, na medida em que se valorizam capacidades, autoestima, autoconfiança e autorrealização ${ }^{6}$.

Para que o processo educativo seja eficaz e eficiente, recomenda-se que seja problematizador, no qual o educador considere o saber do educando para, a partir daí, construir um conhecimento em que o sujeito do aprendizado compreenda seu protagonismo no processo e sinta-se motivado para a mudança de condutas, ampliando seu conhecimento e transformando comportamentos prejudiciais ou inadequados ${ }^{7}$.

O processo educativo desenvolvido com a pessoa com estomia é fundamental para que alcance autonomia no seu cuidado, para favorecer a inclusão social e para evitar complicações tanto na estomia e pele periestoma quanto comprometimentos sociais e psicológicos ${ }^{5}$. Corroborando, a educação em saúde, que visa à orientação para o autocuidado, torna-se fundamental para tais pessoas, principalmente quando se compreende sua importância como sujeito histórico, temporal, criativo e cultural, favorecendo a adaptação à nova condição de saúde ${ }^{8}$.

\section{MÉTODO}

Estudo qualitativo e exploratório, apoiado no materialismo histórico e no método dialético. Foi desenvolvido em um Centro Municipal de Reabilitação, no Rio de Janeiro, onde se desenvolve o Programa de Atenção à Pessoa com estomia. 
O materialismo histórico, que fundamenta o método dialético, preconiza uma análise que considera os contextos histórico, social, político, cultural em que o fenômeno investigado se situa. Busca as contradições que compõem o objeto de estudo, para através da análise de tais contradições, se possa evoluir para uma compreensão mais alargada e aprofundada do objeto. Outrossim, é um método que se ancora no mundo real, no conceito, na teoria e ciência, e com esses elementos é que a análise procede ${ }^{9}$.

Participaram do estudo seis enfermeiros que atuavam neste Centro de Reabilitação e que se adequaram aos seguintes critérios de inclusão: a) enfermeiro que fizesse parte do quadro funcional do Centro Municipal, em regime jurídico único ou por contrato de trabalho; b) enfermeiro que participasse ou já tivesse participado do processo educativo de pessoas com estomia, atendidas na instituição; c) enfermeiro que atuasse com pessoas com estomia há mais de seis meses, possuindo experiência no processo educativo desta clientela. O critério de exclusão selecionado foi: enfermeiro que estivesse de férias ou licenciado no momento da coleta de dados.

Destaca-se que, à época da coleta de dados, existia no quadro do Programa sete enfermeiros, um deles era o coordenador de enfermagem, mas que anteriormente já havia desenvolvido orientações para o autocuidado às pessoas com estomia, e seis enfermeiros atuavam diretamente na consulta de enfermagem. Dentre os sete enfermeiros que estavam entre os critérios de seleção, um se recusou participar do estudo, alegando motivos pessoais.

Para o procedimento de coleta de dados, o qual foi realizado entre dezembro de 2014 e março de 2015, adotouse a triangulação do método, utilizando-se a entrevista semiestruturada individual com os enfermeiros, a observação participante das consultas de enfermagem, totalizando 120 horas e, ainda, a análise dos registros dos enfermeiros nos prontuários das pessoas com estomia. O roteiro da entrevista era composto de três questionamentos básicos: i) discorra como você desenvolve o processo educativo das pessoas com estomia; ii) descreva as ações educativas realizadas com vistas à inclusão social; iii) discorra sobre as facilidades e as dificuldades para o desenvolvimento do processo educativo com pessoas com estomias.

A pesquisa foi aprovada pelo Comitê de Ética em Pesquisa, com número de CAAE 36520814.0.0000.5282 e a coleta dos dados ocorreu entre os meses de dezembro de 2014 a março de 2015. Os participantes receberam o Termo de Consentimento Livre e Esclarecido e foram representados pela letra $E$, seguida por algarismos arábicos na ordem em que as entrevistas ocorreram, atendendo às recomendações da Resolução no 466/2012, do Conselho Nacional de Saúde ${ }^{10}$.

As informações foram tratadas à luz do método dialético, cuja aplicabilidade visou a compreensão das práticas e vivências dos participantes em relação ao processo educativo dirigidos às pessoas com estomias. Assim, a realidade estudada expressou-se nos diálogos dos participantes, por meio da escrita e da linguagem verbal e não verbal, captadas a partir das técnicas de observação participante, entrevistas e análise documental.

A aplicação deste método fez emergir a seguinte categoria analítica: Processo educativo e os paradoxos entre organização laboral, processo de formação e cotidiano do enfermeiro.

\section{RESULTADOS E DISCUSSÃo}

\section{Processo educativo e os paradoxos entre organização laboral, processo de formação e cotidiano do enfermeiro}

A análise dos dados revelou que muitas das dificuldades percebidas pelos enfermeiros durante a execução do processo educativo, visando o autocuidado de pessoas com estomia, estiveram atreladas à configuração da organização do trabalho, especialmente relacionadas aos recursos materiais e a estrutura física do campo. Porém, também foram elencados aspectos dificultadores vinculados à formação profissional e à complexidade da atenção à pessoa com estomia.

A utilização de recursos audiovisuais e materiais didáticos é importante para o processo educativo, por torná-lo dinâmico e por favorecer uma linguagem informal. Porém, a instituição não contava com computadores, não possuía prospectos explicativos para serem fornecidos, e não detinha recursos financeiros para impressão e cópia de materiais diversos. Apresentava apenas dois quadros explicativos nos consultórios, utilizados por alguns enfermeiros durante o processo de ensino-aprendizagem.

Uma dificuldade é a falta de computadores e internet, temos que contar com recursos próprios. Como é que a gente vai fornecer um folder se não tem dinheiro nem para as outras xerox? Além disso, falta material educativo, não há praticamente nada aqui. Estas são dificuldades importantes para realizarmos uma orientação adequada (E1)

Falta material educativo! Foi a primeira coisa que eu cheguei aqui e senti falta. Sem contar que muitas vezes não temos verbas para tirar cópias de nosso material de trabalho, como as fichas admissionais, e isso dificulta muito (E6).

A carência de recursos materiais e audiovisuais, muitas vezes indispensáveis para o processo educativo, torna-se entrave para a realização do trabalho do educador, desmotivando-o e impulsionando-o a adaptar e improvisar recursos 
disponíveis. Essa carência reflete o pouco investimento da organização do trabalho em fornecer condições adequadas para o bom desenvolvimento do processo laboral. Situação que tem sido frequente no setor saúde por conta da aplicação do ideário neoliberal neste contexto, determinando entre outras consequências, o desinvestimento em infraestrutura material e na força de trabalho ${ }^{11}$.

De outro modo, assevera-se que a escassez de recursos audiovisuais não inviabiliza o processo educativo, pois nada substitui a relação dialógica entre os envolvidos. O estabelecimento do diálogo entre o enfermeiro e a clientela promove a troca de conhecimentos, podendo propiciar a aquisição de soluções para as dificuldades apresentadas durante a aprendizagem ${ }^{12}$. Corrobora-se que é necessário, além de recursos audiovisuais, a confiança e as boas relações interpessoais para que o processo educativo seja desenvolvido adequadamente, favorecendo a apreensão de conteúdos necessários para o autocuidado, tornando assim, o processo educativo mais acolhedor, dinâmico e criativo para a clientela ${ }^{12}$.

Destaca-se que, durante a observação participante nas consultas de enfermagem, verificou-se que muitos enfermeiros utilizavam aparelhos eletrônicos pessoais para mostrarem figuras e vídeos sobre os cuidados com a estomia e reproduziam impressos com recursos próprios, a fim de favorecer um processo educativo mais dinâmico e atrativo.

Outro aspecto que dificultava o processo educativo das pessoas com estomia era o fato de os técnicos de enfermagem não desempenharem funções adequadas ao seu cargo, não auxiliando, assim, o enfermeiro nos procedimentos necessários, além do alto índice de absenteísmo e afastamentos, comprometendo o trabalho do enfermeiro:

Eu não tenho um técnico de enfermagem para me auxiliar nos curativos, ou qualquer outra atribuição com o paciente. Não preciso de um técnico de enfermagem para preencher papel. Para isso, poderíamos ter um auxiliar administrativo. Eu acabo tendo que fazer o papel deles em muitos momentos, o que atrapalha o andamento do meu trabalho (E1).

Às vezes falta um profissional de enfermagem, um técnico de enfermagem para fazer o acolhimento e até mesmo pegar um prontuário. Às vezes temos que parar e ir pegar o prontuário, o que dificulta um pouco. Não que a gente não queira pegar, mas às vezes você chega ali e tem outros clientes aguardando atendimento, este desvio de função acaba atrasando as consultas de enfermagem, prejudicando o meu trabalho e o atendimento das pessoas estomizadas (E5).

Desta forma, o enfermeiro acabava realizando as atividades de responsabilidade dos técnicos de enfermagem, diminuindo seu tempo disponível para as consultas e para o processo educativo.

A enfermagem convive historicamente com pouca valorização e baixo reconhecimento de seu trabalho, em especial no que diz respeito às condições de trabalho, remuneração e autonomia profissional, enfraquecendo a luta pelos seus direitos trabalhistas e por melhores condições de trabalho, e acabam realizando múltiplas tarefas que não são de sua responsabilidade, levando-os ao estresse e diminuindo até mesmo a qualidade da assistência desenvolvida com a clientela ${ }^{13-14}$.

Nesse âmbito, também se captou importante contradição, pois, segundo a Lei no $7.498 / 86^{15}$, que dispõe sobre a regulamentação do exercício profissional da enfermagem, os enfermeiros devem exercer privativamente o planejamento, organização, coordenação, execução e avaliação dos serviços de assistência de enfermagem. CabendoIhes ainda liderar e avaliar toda a equipe de enfermagem.

Assim, a partir das falas selecionadas anteriormente, depreende-se que há equívocos na liderança dos participantes, pois é papel do enfermeiro determinar as ações a serem desenvolvidas pelos técnicos de enfermagem, considerando as habilidades e competência dos membros da equipe de enfermagem.

A escassez ou inexistência de alguns materiais, como equipamentos coletores e adjuvantes, representavam um entrave para a qualidade do processo educativo dos clientes com estomia, dificultando o processo de autocuidado e inclusão social desta clientela:

A dificuldade é quando começa a faltar o equipamento, pois o paciente chega muito ansioso, querendo aquele material que ele já está adaptado e, muitas vezes, não tem. Então, recomeça toda readaptação a um novo material, novas orientações e mais processo educativo. Às vezes, não tem equipamentos de uma peça, por falta do processo de licitação, ou por atraso, às vezes o material que tem não é adequado para aquele paciente. Difícil! (E5).

A ausência dos equipamentos e outras tecnologias levam as pessoas com estomia a passarem por um novo processo de adaptação a sua condição de saúde, como também resulta na retomada do processo educativo empreendido pelos enfermeiros, obstaculizando o autocuidado e a inclusão social destas pessoas ${ }^{5}$.

Assim, evidenciou-se outra contradição, pois uma instituição de referência no atendimento às pessoas com estomia deve garantir e contar com diversificados tipos de equipamentos e outras tecnologias para fornecimento aos 
clientes, considerando as especificidades do tipo de pele, classificação da estomia, atividades laborais e de lazer que desenvolve, entre outros aspectos ${ }^{5}$.

Ademais, a Portaria no 400/200916 determina que as pessoas com estomia tenham o fornecimento mensal de equipamentos adequados e de adjuvantes para que possam ter vida digna e evitar complicações.

O espaço físico inadequado também foi aludido nas falas dos enfermeiros como um problema da unidade:

O que dificulta, no caso do meu trabalho, é o espaço que não é bom, aqui na parte da dispensação. Isso não interfere muito com o paciente, é um problema mais interno, que compromete o nosso trabalho e a nossa saúde mental (E4).

A estrutura física tem que melhorar, pois dificulta o nosso trabalho, afeta o bom andamento do serviço e nos estressa. Há falta de um banheiro maior e adaptado aos clientes. Dificulta o ensino da auto irrigação, dificulta a mobilização e dificulta (E6).

Verificou-se, através das observações participantes, que o local contava realmente com uma estrutura física inadequada, como por exemplo, cita-se uma copa inserida em um pequeno almoxarifado, que continha alguns equipamentos e adjuvantes. Com base na Norma Regulamentadora $n^{0} 32$ (NR32) ${ }^{17}$, o recomendado seria uma copa independente para realização de pequenas refeições, para que não fossem consumidos alimentos próximo à área de armazenamento de materiais e dos postos de trabalho, o que é proibido legalmente.

A estrutura física inadequada ocorria também no setor de consultas de enfermagem. Não existia uma sala de reuniões e nem espaço para desenvolvimento de grupos de apoio e acolhimento as pessoas com estomia, contrariando o preconizado pela Portaria no 400/2009¹6

Outro fator percebido pelos participantes e que gerava dificuldade para o desenvolvimento do processo educativo, relacionava-se ao escasso conteúdo teórico-prático desenvolvido no ensino da graduação ligado à área da Estomaterapia. Ademais, de acordo com os participantes, os cursos de especialização estavam centrados no modelo biomédico de ensino e assistência, pouco privilegiando a dimensão psicossocial do cliente e família.

Temos uma visão limitada na própria formação da graduação e pós-graduação sobre conteúdos de Estomaterapia. Não nos dão essa direção relacionada à inclusão social, e muito menos para preparar para o trabalho, para o lazer, para a sexualidade destas pessoas (E1).

A parte de graduação, não sei como está hoje, mas eu não vi nada de estomia. Na pós-graduação, eu também não tive um conteúdo mais integral do cuidado. A gente fica bem focado na parte física. (E4).

Estas falas estão também relacionadas com o perfil dos participantes do estudo, cuja maioria era formada há mais de vinte anos, um período em que o ensino de graduação se caracterizava predominantemente biomédico no país ${ }^{18}$. Ademais, a análise documental também corroborou estas inferências, ao identificar nos registros dos enfermeiros que todos estavam voltados para orientações e cuidados direcionados à dimensão fisiológica da pessoa com estomia, e nenhum registro continha dados relacionados às necessidades psicossociais da clientela.

É interessante verificar as contradições existentes nesta situação, pois os próprios enfermeiros reconheciam as limitações no processo de formação, mas continuavam direcionando seus atendimentos à parte fisiológica, não abordando questões relacionadas às dimensões psicossociais da clientela e, assim, obstaculizando uma inclusão social abrangente e fortalecida.

Emergiram também elementos que poderiam facilitar o processo educativo junto às pessoas com estomias, como a formação de grupos de apoio, pois possibilita o acolhimento entre os pares e a troca da experiência vivida.

Eu acho que o que pode complementar esta parte educativa são as reuniões em grupo, que facilitam muito a adaptação dos estomizados, pois ali eles compreendem que não estão sozinhos e trocam experiências importantes sobre o dia a dia (E3).

Outro projeto paralelo que está se desenvolvendo é o grupo de apoio, que já era para eu ter feito em janeiro, e acabou não dando, pois ele contribui muito para o processo de ensino-aprendizagem dos clientes $e$ familiares (E6).

A participação das pessoas em grupos de apoio favorece a aceitação da condição de estar com uma estomia, além de despertar o interesse pela luta por seus direitos enquanto cidadãos e pessoas com deficiência, incentivando e fortalecendo ações por maior inclusão social. O diálogo é a base deste grupo, e sua dinâmica operacional contrapõe-se ao modelo biomédico, por abordar temáticas que vão além dos cuidados à estomia e pele periestoma, abrangendo questões sobre a integralidade do ser humano ${ }^{19}$.

Também foi citado como facilitador do processo educativo o fato de o Programa possuir variedade de equipamentos coletores e adjuvantes para fornecimento aos clientes com estomia. 
A gente tem uma variedade grande de equipamentos, cada vez mais o mercado está lançando materiais e a gente tenta colocar isso aqui no Programa, então acho que a gente tem isso, e facilita bem (E4).

Nessa fala também se evidenciou uma contradição, pois, durante as observações de campo e na análise das falas de outros participantes, apreendeu-se falta e inadequação de equipamentos e adjuvantes no Programa. Compreendese que a percepção deste enfermeiro estava centrada também na comparação do Programa com outros polos de atendimento, que não fornecem aos usuários a diversidade de materiais oferecidos por este Centro de reabilitação.

\section{CONCLUSÃO}

Verificou-se que houve mais fatores dificultadores que facilitadores do processo educativo de pessoas com estomia no campo investigado. Assim, as dificuldades apreendidas foram em relação à inadequação da estrutura física e à escassez de recursos financeiros, materiais e humanos. Além desses fatores, considerou-se que a formação e a qualificação dos enfermeiros são elementos de entrave para um processo educativo que visem um autocuidado abrangente, por estarem fundamentados em conteúdo puramente biomédico, não incluindo orientações que visassem as necessidades psicossociais da clientela.

Em relação aos aspectos facilitadores, constatou-se que estiveram relacionados ao desenvolvimento de grupos de apoio e acolhimento às pessoas com estomia. Também se aprendeu que a disponibilidade e fornecimento de tecnologias do cuidado em Estomaterapia foi considerado um fator positivo para o bom desenvolvimento do processo educativo.

A partir dos dados analisados, evidenciou-se contradições e discrepâncias na execução do processo educativo, destacando-se a percepção de que a formação é um entrave para o bom desenvolvimento deste processo, porém os participantes permanecem focados em orientações que visam a dimensão física das pessoas com estomias. Outra contradição envolve a organização do trabalho, que não fornece insumos adequados em termos quantitativos e qualitativos para que o processo de orientação para o autocuidado de pessoas com estomia ocorra a contento.

Também se verificou contradições referente ao cumprimento de aspectos legais que respaldam o desenvolvimento do autocuidado de pessoas com estomia. Assim, há o amparo jurídico para assegurar e fazer cumprir o fornecimento de equipamentos coletores e adjuvantes para o cuidado a estas pessoas, mas é descumprido pela organização do trabalho.

\section{REFERÊNCIAS}

1. Santos CS, Araújo LS, Paraguai LN, Aoyama EA, Lima RN. Nursing care for colostomy patients. ReBIS [internet], 2020 [cited 2020 May 01]; 2(1):27-33. Available from: http://revista.rebis.com.br/index.php/rebis/article/view/332.

2. Souza MMT, Moraes AA, Balbino CM, Silvino ZR, Tavares CMM, Passos JP. Emotional support provided by the nurse to the ostomized patient. Revista Portuguesa de Enfermagem de Saúde Mental [Internet], Porto, 2016 [cited 2020 May 01]; (spe4):4956. DOI: https://doi.org/10.19131/rpesm.0141.

3. Moreira LR, Souza JC, Oliveira MM, Melo NS, Cerqueira TF. Self care with ostomy: understanding of hospitalized patients about the orientations received by staff. Enfermagem Revista [internet], [cited 2020 May 01]; 20(2):116-134. Available from: http://periodicos.pucminas.br/index.php/enfermagemrevista/article/view/16329.

4. Ministério da Saúde (BR). Instituto Nacional de Câncer José Alencar Gomes da Silva (INCA). Coordenação Geral de Ações Estratégicas. Coordenação de Prevenção e Vigilância. Estimativa 2020: incidência de câncer no Brasil. Rio de Janeiro: Ed. INCA, 2020 [cited 2020 May 01]; Available from: https://www.inca.gov.br/publicacoes/livros/estimativa-2020-incidencia-de-cancerno-brasil.

5. Carvalho DS, Silva AGI, Ferreira SRM, Braga LC. Elaboration of na educational technology for ostomized patients: peristomal skin care. Rev. Bras. Enferm. [Internet], Brasília, 2019 [cited 2020 May 01]; 72(2):427-34. DOI: https://doi.org/10.1590/0034-71672016-0024.

6. Mauricio VC. A pessoa estomizada e o processo de inclusão no trabalho: contribuições para enfermagem [master thesis]. Rio de Janeiro (RJ): Universidade do Estado do Rio de Janeiro [cited 2020 May 01]; 2011.

7. Rosado SR, Cicarini WB, Filipini CB, Lima RS, Dázio EMR. Educational health practices developed by nurses for people with ostomy. Enfermagem Brasil [Internet], 2015 [cited 2020 May 01]; 14(4):235-41. Available from: http://portalatlanticaeditora.com.br/index.php/enfermagembrasil/article/viewFile/47/53.

8. Mauricio VC. Processo educativo desenvolvido por enfermeiros voltado para inclusão laboral de pessoas com estomia [doctoral dissertation]. Rio de Janeiro (RJ): Universidade do Estado do Rio de Janeiro, 2015 [cited 2020 May 01].

9. Martins LM, Lavoura TN. Historical-dialectical materialism: contributions to qualitative research in education. Educ. rev. [internet], Curitiba, 2018 [cited 2020 May 01]; 34(71):223-39. DOI: https://doi.org/10.1590/0104-4060.59428.

10. Ministério da Saúde (BR). Conselho Nacional de Saúde. Resolução no. 466, de 12 de Dezembro de 2012. Diretrizes e normas regulamentadoras de pesquisas envolvendo seres humanos. Brasília (DF): MS, 2012 [cited 2020 May 01]; Available from: https://conselho.saude.gov.br/resolucoes/2012/Reso466.pdf. 
11. Souza NVDO, Gonçalves FGA, Pires AS, David HMSL. Neoliberalist influences on nursing hospital work process and organization. Rev. Bras. Enferm. [Internet], Brasília, 2017 [cited 2020 May 01]; 70(5):912-9. DOI: https://doi.org/10.1590/0034-7167-20160092.

12. Barros MMAF, Mendes MLC, Frota LMA, Almeida JRS. User embracement in a primary health center: potentialities and challenges. SANARE, Sobral [internet], 2018 [cited 2020 May 01]; 17(2):114-9. DOI: https://doi.org/10.36925/sanare.v17i2.1269.

13. Lage CEB, Alves MS. Evaluation of nrusing and implications in nurses dayly routine. Enferm. Foco [internet], 2016 [cited 2020 May 01]; 7(3/4):12-6. DOI: https://doi.org/10.21675/2357-707X.2016.v7.n3/4.908.

14. Lorenzetti J, et al. Unidade de ação: um desafio para a enfermagem brasileira. Enfermagem em foco [Internet], 2012 [cited 2019 Mar 15]; 3(3):152-4. DOI: https://doi.org/10.21675/2357-707X.2012.v3.n3.304.

15. Brasil. Lei no 7.498, de 25 de junho de 1986. Dispõe sobre a regulamentação do exercício da enfermagem, e dá outras providências. Diário Oficial [da] República Federativa do Brasil 26 jun 1986 [cited 2020 May 01]; Seção I: 9273-5. Available from: http://www.cofen.gov.br/lei-n-749886-de-25-de-junho-de-1986_4161.html.

16. Ministério da Saúde (BR). Portaria no 400, de 16 de novembro de 2009. Estabelece Diretrizes Nacionais para a Atenção à Saúde das Pessoas Ostomizadas no âmbito do Sistema Único de Saúde - SUS. Diário Oficial [da] República Federativa do Brasil 16 nov 2009 [cited 2020 May 01]; Seção I: 41-2. Available from: http://bvsms.saude.gov.br/bvs/saudelegis/sas/2009/prt0400_16_11_2009.html.

17. Ministério do Trabalho e Emprego (BR). Portaria no. 485, de 11 de novembro de 2005. NR 32 - Segurança e Saúde no Trabalho em Serviços de Saúde. Diário Oficial [da] República Federativa do Brasil, Brasília, DF, 16 nov 2005. [cited 2020 May 01]; Available from: https://www20.anvisa.gov.br/segurancadopaciente/index.php/legislacao/item/portaria-n-485-de-11-denovembro-de-2005.

18. Martins C, Kobayashi RM, Ayoub AC, Leite MMJ. Profile of the nurse and the necessities of professional competence development. Texto \& contexto - enferm. [internet], Florianópolis, 2006 [cited 2020 May 01]; 15(3). DOI: https://doi.org/10.1590/S0104-07072006000300012.

19. Souza LRG, Teixeira NO, Silva RAR, Porfirio RBM. Nursing care for ostomized patient: literature review. REVA Acad. Rev. Cient. da Saúde [internet], 2020 [cited 2020 May 01]; 5(1):18-27. DOI: https://doi.org/10.24118/reva1806.9495.5.1.2020.663. 